\title{
Differential effects of $\beta$-arrestin 1 and $\beta$-arrestin 2 on somatostatin receptors in murine AtT-20 corticotroph tumor cells
}

\author{
Kazunori Kageyama, Rie Hagiwara, Kanako Niioka, Shinobu Takayasu and Makoto Daimon \\ Department of Endocrinology and Metabolism, Hirosaki University Graduate School of Medicine, 5 Zaifu-cho, Hirosaki, Aomori \\ 036-8562, Japan
}

\begin{abstract}
Autonomous production of adrenocorticotropic hormone (ACTH) from pituitary corticotroph adenomas is the primary cause of Cushing's disease. Somatostatin receptor, a G protein-coupled receptor (GPCR), types 2 (SSTR2) and 5 (SSTR5) mRNA expression is greater than that of other SSTR subtypes in human corticotroph adenomas. Further, the multiligand SOM230 shows potent effects in decreasing ACTH plasma levels and urinary free cortisol levels in patients with Cushing's disease. We previously showed that both Sstr2 and Sstr 5 mRNA levels were unaffected by SOM230 treatment, suggesting that both receptors might not be downregulated by the agonist. Intracellular molecules, such as $\beta$-arrestins, modulate ligand activated-receptor responses. In the present study, we determined regulation of $\beta$-arrestin 1 and $\beta$-arrestin 2 by SOM230 and dexamethasone in murine AtT-20 corticotroph tumor cells. In addition, we examined the effects of $\beta$-arrestin1 and $\beta$-arrestin 2 on Sstr mRNA and their protein levels. SOM230 treatment increased $\beta$-arrestin1 mRNA levels and did not alter $\beta$-arrestin 2 mRNA levels. SOM230 treatment could induce $\beta$-arrestin1 production in corticotroph tumor cells. Dexamethasone treatment decreased $\beta$-arrestin 2 mRNA levels. $\beta$-arrestin 2 knockdown increased proopiomelanocortin, and both Sstr2 and Sstr 5 mRNA and their protein levels. The $\beta$-arrestin2 knockdown-increased proopiomelanocortin mRNA levels were canceled by SOM230 treatment.
\end{abstract}

Key words: Somatostatin, ACTH, Corticotroph, Arrestin

\begin{abstract}
AMONG G PROTEIN-COUPLED RECEPTOR (GPCR)-TARGETING DRUGS, somatostatin receptor agonists have demonstrated inhibitory effects on tumor cell proliferation in pituitary tumors. Octreotide, which is used for somatotroph and thyrotroph tumors, is a somatostatin receptor type 2 (SSTR2)-preferring somatostatin analogue [1]. The multiligand SOM230 (or pasireotide) binds with high affinity to SSTR1, 2, 3, and 5, and displays a 30- to 40-fold higher affinity for SSTR1 and SSTR5 than octreotide [2]. In human corticotroph adenomas, SSTR 2 and SSTR 5 mRNA expression surpasses that of other SSTR subtypes [3]. Therefore, in patients with Cushing's disease, SOM230 exhibits the potent ability to decrease adrenocorticotropic hormone (ACTH) plasma levels and urinary free cortisol level $[4,5]$.

Intracellular molecules, such as $\beta$-arrestins, contribute
\end{abstract}

Submitted Apr. 30, 2020; Accepted Aug. 28, 2020 as EJ20-0251

Released online in J-STAGE as advance publication Sep. 18, 2020

Correspondence to: Kazunori Kageyama, M.D., Ph.D., Department of Endocrinology and Metabolism, Hirosaki University Graduate School of Medicine, 5 Zaifu-cho, Hirosaki, Aomori 036-8562, Japan.

E-mail: kkageyama@hkg.odn.ne.jp to membrane receptor phosphorylation, desensitization, and trafficking of GPCRs, thereby modulating ligand activated-receptor responses [6]. $\beta$-arrestins regulate internalization and recycling of SSTRs [7]. Further, low $\beta$-arrestin expression correlates with responsiveness to somatostatin analogues [8].

In the present study, we explored the regulation of $\beta$ arrestin 1 and $\beta$-arrestin 2 by SOM 230 and dexamethasone in murine AtT-20 cells. To elucidate further roles of $\beta$-arrestin1 and $\beta$-arrestin2, we then examined for the first time the effects of $\beta$-arrestin1 and $\beta$-arrestin2 on Sstr mRNA levels and proopiomelanocortin (Pomc), an ACTH precursor gene.

\section{Materials and Methods}

\section{Materials}

SOM230 was kindly provided by Novartis Pharma AG (Basel, Switzerland). Dexamethasone was purchased from Sigma-Aldrich Corp. (St. Lois, MO, USA).

\section{Cell culture}

Murine AtT-20 pituitary corticotroph tumor cells were 
obtained from ATCC (Manassas, VA, USA). The cells were cultured in Dulbecco's modified Eagle's medium (DMEM) (Sigma-Aldrich Corp.) supplemented with $10 \%$ fetal bovine serum (FBS), $100 \mu \mathrm{g} / \mathrm{mL}$ streptomycin, and $100 \mathrm{U} / \mathrm{mL}$ penicillin at $37^{\circ} \mathrm{C}$ in a humidified atmosphere $\left(5 \% \mathrm{CO}_{2}\right.$ and $95 \%$ air). The cells were plated in 6 well plates at $15.0 \times 10^{4}$ cells $/ \mathrm{cm}^{2}$ for 2 days before each experiment and the medium was changed every $48 \mathrm{~h}$. On day 3 , to remove the effect of factors contained in FBS, the cells were washed, then starved overnight with DMEM supplemented with $0.2 \%$ bovine serum albumin (BSA) prior to each experiment. At the end of each experiment, total cellular RNA was collected and stored at $-80^{\circ} \mathrm{C}$ until the relevant assay was performed.

\section{$R N A$ extraction}

The cells were incubated with medium alone (control) or medium containing SOM230 or dexamethasone for the indicated times. Additionally, to examine the dosedependent effects of SOM230 or dexamethasone, cells were incubated with medium alone (control) or medium containing increasing concentrations (1-100 nM) of SOM230 or dexamethasone. At the end of each experiment, total cellular RNA was extracted using the RNeasy Mini Kit (QIAGEN, Hilden, Germany) according to the manufacturer's protocol. cDNA was synthesized from total RNA $(0.5 \mu \mathrm{g})$ using random hexamers as primers with the SuperScript First-Strand Synthesis System for Reverse Transcriptase-Polymerase Chain Reaction (RTPCR) (Invitrogen Corp., Carlsbad, CA, USA) according to the manufacturer's instructions.

\section{Real-time RT-PCR}

Total cellular RNA extraction and cDNA synthesis were performed as described previously [9]. The resulting cDNA was then subjected to real-time PCR as follows. The mRNA expression levels of mouse $\beta$ arrestin1/2, Pomc, and Sstr2/5 were evaluated using quantitative real-time PCR with specific sets of primers and probes (Assays-on-Demand Gene Expression Products; Applied Biosystems, Foster City, CA, USA). $\beta 2$-microglobulin $(B 2 \mathrm{mg})$ was used as a housekeeping gene to standardize expression levels.

Each reaction consisted of $1 \times$ TaqMan Universal PCR Master Mix (Applied Biosystems), $1 \times$ Assays-on-Demand Gene Expression Products (Mm00617540_m1 for mouse $\beta$-arrestin1, Mm00520666 g1 for mouse $\beta$-arrestin2, Mm00435874_m1 for mouse Pomc, Mm00436685_g1 for mouse Sstr2, Mm01307775_s1 for mouse Sstr5, and Mm00437762_m1 for mouse B2 $\mathrm{mg}$ ), and $500 \mathrm{ng}$ cDNA (25 $\mu \mathrm{L}$ total volume) with the following parameters on an ABI PRISM 7000 Sequence Detection System (Applied Biosystems): $95^{\circ} \mathrm{C}$ for $10 \mathrm{~min}, 40$ cycles at $95^{\circ} \mathrm{C}$ for
$15 \mathrm{~s}$, and $60^{\circ} \mathrm{C}$ for $1 \mathrm{~min}$. All data are expressed as a function of the threshold cycle $\left(\mathrm{C}_{\mathrm{T}}\right)$ for quantitative analyses using ABI PRISM 7000 SDS software (Applied Biosystems).

\section{$\boldsymbol{R} \mathbf{A} \boldsymbol{A}$ interference experiments}

$\beta$-arrestin $1 / 2$ and control small interfering RNAs (siRNAs) were designed and purchased from QIAGEN. The cells were transfected with siRNA and HiPerFect transfection reagent (QIAGEN) according to the manufacturer's protocol. The cells were seeded into 6-well plates at a density of $10 \times 10^{4}$ cells/well, incubated for $24 \mathrm{~h}$ in culture medium $(1 \mathrm{~mL})$ containing siRNA for either control (siControl) or $\beta$-arrestin1/2 (siArrb1, Mm_Arrb1_6; siArrb2, Mm_Arrb2_1), and then incubated for $24 \mathrm{~h}$ further. To examine the effects of SOM230 or dexamethasone, cells were starved overnight with DMEM supplemented with $0.2 \%$ BSA, and then incubated with medium alone (control) or medium containing increasing $100 \mathrm{nM}$ of SOM230 for $6 \mathrm{~h}$ or dexamethasone for $24 \mathrm{~h}$. The expression of Pomc, Sstr2/5, and $B 2 m g$ mRNA was examined by quantitative RTPCR. The expression of SSTR2/5, and $\beta$-actin protein was examined by Western blot analysis.

\section{Western blot analysis}

Cells were washed with cold phosphate-buffered saline (PBS), and were lysed with Laemmli sample buffer for protein extraction. The lysates were centrifuged, and the supernatant was recovered. Western blot analysis was performed as described previously [9]. In brief, whole cell lysates were heated, and proteins were separated on a $2-15 \%$ gradient polyacrylamide gel and transferred to a polyvinylidene fluoride membrane (Daiichi Kagaku, Tokyo, Japan). Membranes blocked with Detector Block ${ }^{\circledR}$ buffer (Kirkegaard \& Perry Laboratories, Gaithersburg, MD) were incubated for $1 \mathrm{~h}$ with an anti-SSTR2 antibody (1:1,000 dilution) (Santa Cruz Biotechnology, Dallas, TX, USA), anti-SSTR5 antibody (1:5,000 dilution) (Proteintech Group, Rosemont, IL, USA) and anti- $\beta$-actin antibody (1:10,000 dilution) (ab8227, Abcam, Cambridge, MA, USA). After washes with PBS containing $0.05 \%$ Tween 20 , the membrane was incubated with horseradish peroxidase-labeled anti-mouse (Santa Cruz Biotechnology) or anti-rabbit (Daiichi Kagaku) immunoglobulin G. Hybridization signals were detected by the chemiluminescent substrate SuperSignal West Pico (Pierce Chemical Co., Rockford, IL, USA), and the membrane was exposed to BioMax film (Eastman Kodak Co., Rochester, NY, USA).

\section{ACTH assays}

AtT-20 cells were treated with $100 \mathrm{nM}$ of SOM230 
for $24 \mathrm{~h}$, and the supernatant of the culture medium was collected and stored at $-80^{\circ} \mathrm{C}$ until assay. The ACTH concentrations in the culture media were measured with an ACTH enzyme-linked immunosorbent assay (ELISA) kit (MD Bioproducts, Zurich, Switzerland).

\section{Statistical analysis}

Each in vitro experiment was performed at least three times. Samples were used in triplicate for each group of experiments. Each value is expressed as the mean \pm standard error of the mean. Statistical analysis was performed with analysis of variance (ANOVA), followed by a Fisher's protected least-significant difference post hoc test. The level of statistical significance was set at $p<0.05$.

A

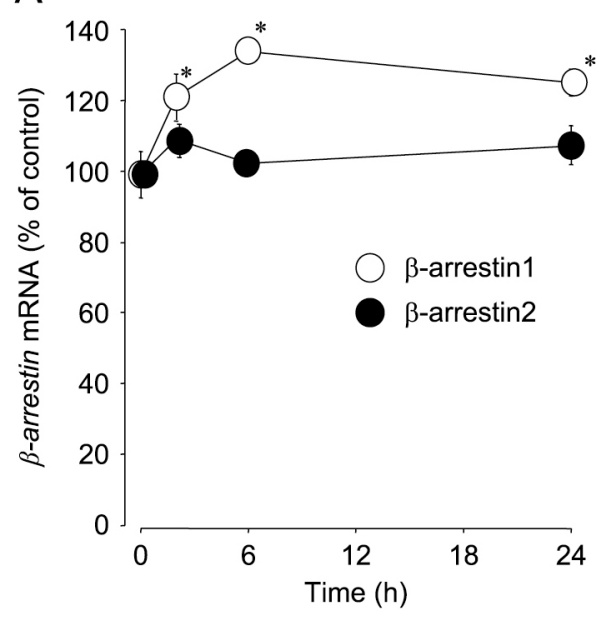

C

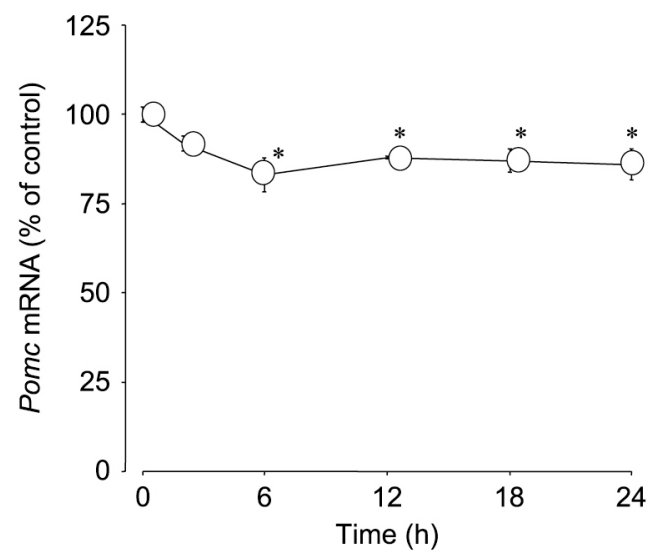

\section{Results}

\section{Effects of SOM230 treatment on $\beta$-arrestin 1/2 mRNA levels}

A time course study showed that $100 \mathrm{nM}$ SOM230 significantly increased $\beta$-arrestin1 $(p<0.01)$, but not $\beta$-arrestin2 levels (Fig. 1A), with significant effects observed from 2 to $24 \mathrm{~h}$ of treatment. $\beta$-arrestin 1 levels were increased in a dose-dependent manner $(p<0.05)$, with significant effects observed from 10 to $100 \mathrm{nM}$ SOM230 (Fig. 1B). A time course study also showed that $100 \mathrm{nM}$ SOM230 significantly decreased Pomc mRNA levels with significant effects observed from 6 to $24 \mathrm{~h}$ of treatment $(p<0.05$, Fig. 1C). Similarly to our previous report [10], ACTH levels were also reduced within $24 \mathrm{~h}$ of the addition of SOM230 $(p<0.05$, Fig. 1D).

\section{B}

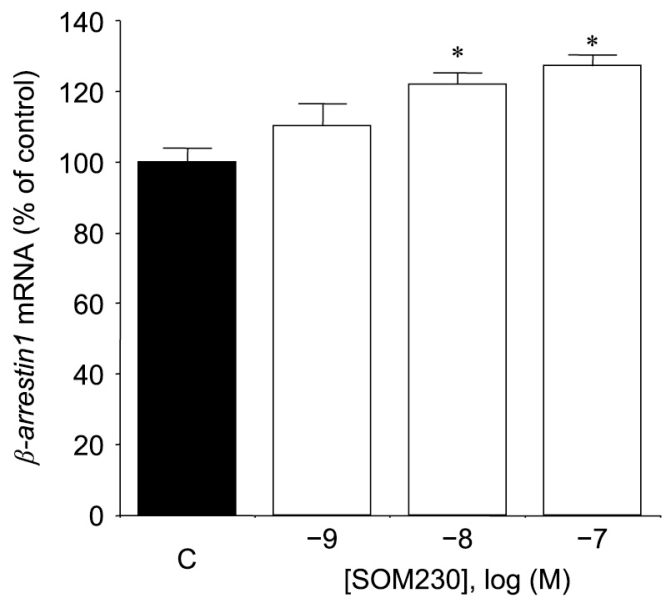

D

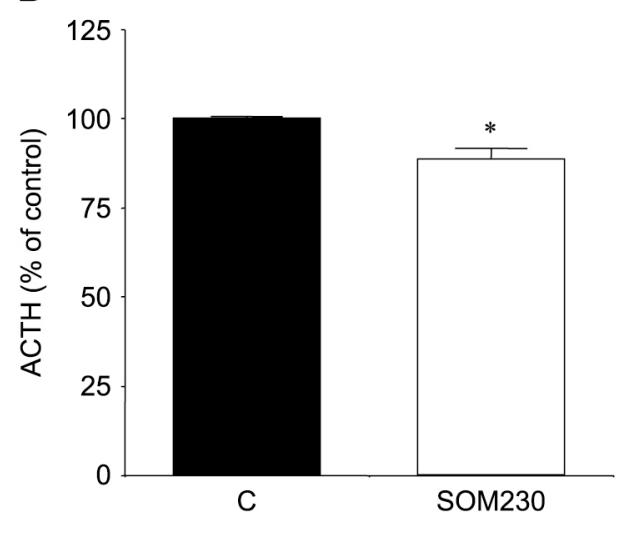

Fig. 1 Effects of SOM230 on $\beta$-arrestin1/2 mRNA levels in murine corticotroph tumor cells. ${ }^{*} p<0.05$ [compared with Time 0 or control (C)]. (A) Time-dependent effects of SOM230 on $\beta$-arrestin1/2 mRNA levels. (B) Dose-dependent effects of SOM230 on $\beta$ arrestin1 mRNA levels. (C) Time-dependent effects of SOM230 on Pomc mRNA levels. (D) Effects of SOM230 on ACTH levels. 


\section{Effects of dexamethasone treatment on $\beta$-arrestin 1/2 mRNA levels}

A time course study showed that $100 \mathrm{nM}$ dexamethasone significantly decreased $\beta$-arrestin $2(p<0.005)$, but not $\beta$-arrestin1 levels (Fig. 2A), with significant effects observed from 2 to $24 \mathrm{~h}$ of treatment. $\beta$-arrestin 2 levels decreased in a dose-dependent manner $(p<0.001)$, with significant effects observed from 1 to $100 \mathrm{nM}$ dexamethasone (Fig. 2B).

\section{Effects of $\beta$-arrestin1/2 on Pomc mRNA levels}

$\beta$-arrestin 1 mRNA levels were reduced by $58 \%$ in cells transfected with siArrb1, but they were increased $(157 \%)$ in cells transfected with siArrb2 (Fig. 3A). $\beta$ arrestin 2 mRNA levels were reduced by $54 \%$ in cells transfected with siArrb2, but they were not changed in cells transfected with siArrb1 (Fig. 3B). $\beta$-arrestin1 knockdown did not exert any significant changes on basal Pomc levels compared with the control, while $\beta$ arrestin 2 knockdown significantly increased basal Pomc levels $(140 \%)(p<0.01)$ (Fig. 3C).

\section{Effects of $\beta$-arrestin1/2 on Sstr2/5 $\mathrm{mRNA}$ and SSTR $2 / 5$ protein levels}

Sstr 2 mRNA levels were significantly increased by $\beta$-arrestin 2 , but not $\beta$-arrestin1, knockdown $(p<0.05)$ (Fig. 4A). Sstr 5 mRNA levels were significantly decreased and increased by $\beta$-arrestin1 and $\beta$-arrestin 2 knockdown, respectively ( $p<0.05$ and $p<0.001$, respectively) (Fig. 4B). SSTR2 protein levels were significantly increased by $\beta$-arrestin2, but not $\beta$-arrestin1, knockdown $(p<0.05)$ (Fig. 4C). SSTR5 protein levels were signifi- cantly increased by $\beta$-arrestin2, but not $\beta$-arrestin1, knockdown $(p<0.005)$ (Fig. 4D).

\section{Effects of $\beta$-arrestin1/2 and SOM230 on Pomc mRNA levels}

SOM230 decreased Pomc mRNA levels in cells transfected with siControl (Fig. 5). Either $\beta$-arrestin1 or $\beta$ arrestin 2 knockdown increased basal Pomc levels (Fig. 5). The $\beta$-arrestin 1 or $\beta$-arrestin 2 knockdown-increased Pomc mRNA levels were canceled by SOM230 treatment (Fig. 5). Compared with each siRNA and SOM230 (-) group (as 100\%), Pomc mRNA levels were decreased to $86 \%$ and $81 \%$ by SOM 230 treatment in cells transfected with siControl or siArrb1, respectively, while Pomc mRNA levels were also further decreased to $73 \%$ by SOM230 in cells transfected with siArrb2.

\section{Effects of $\beta$-arrestin 2 and dexamethasone on $\beta$ - arrestin1, Pomc, and Sstr $5 \mathrm{mRNA}$ levels}

$\beta$-arrestin1 mRNA levels were significantly increased in cells transfected with siArrb2 (Fig. 6A). Similarly, they were also increased in cells transfected with siArrb2 under dexamethasone treatments (Fig. 6A). Pomc mRNA levels were decreased under dexamethasone treatments, while they were increased by $\beta$-arrestin 2 knockdown (Fig. 6B). The $\beta$-arrestin 2 knockdown-induced increases in Pomc mRNA levels were suppressed under dexamethasone treatments (Fig. 6B). Sstr5 mRNA levels were significantly increased by $\beta$-arrestin 2 knockdown (Fig. 6C). The $\beta$-arrestin 2 knockdown-induced Sstr 5 mRNA levels were also inhibited under dexamethasone treatments (Fig. 6C).
A

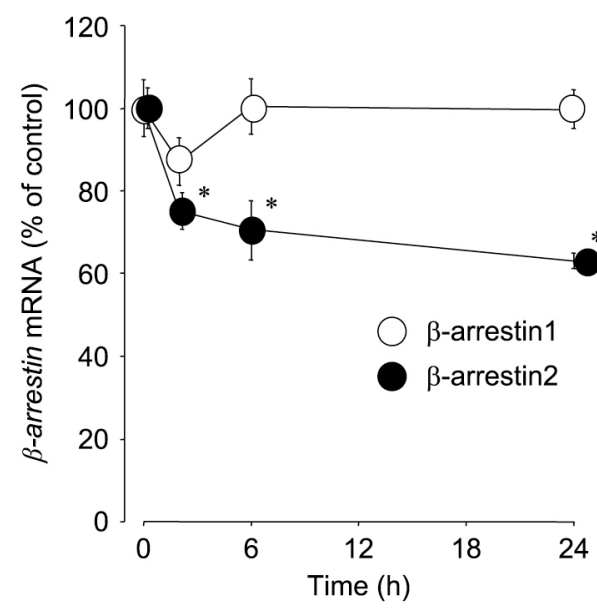

B

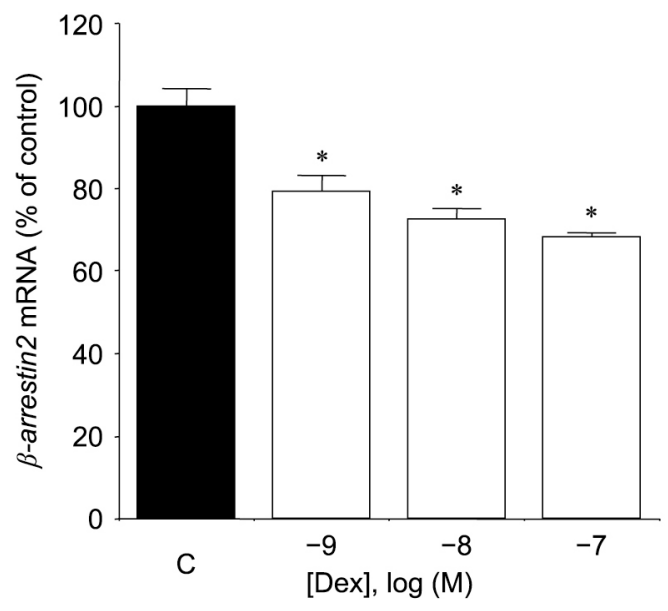

Fig. 2 Effects of dexamethasone on $\beta$-arrestin 1/2 mRNA levels in murine corticotroph tumor cells. $* p<0.05$ [compared with Time 0 or control (C)]. (A) Time-dependent effects of dexamethasone on $\beta$-arrestin1/2 mRNA levels. (B) Dose-dependent effects of dexamethasone on $\beta$-arrestin 2 mRNA levels. 


\section{Discussion}

SOM230 has been used in the treatment of Cushing's disease. SSTR 2 and SSTR 5 mRNA is expressed more frequently than other SSTR subtypes in human corticotroph adenomas [3]. In AtT-20 cells, Sstr 5 mRNA is expressed at much higher levels than other Sstr subtypes [11]. Agonists modulate the expression levels or sensitivity of the receptors [12]. Among SSTRs, SSTR3 is rapidly downregulated by agonists [13]. In our previous study, both Sstr2 and Sstr 5 mRNA levels are unchanged by the presence of SOM230 [10], suggesting that both receptors might not be downregulated by the agonist.

$\beta$-arrestin1 mRNA levels were increased by $\beta$ arrestin2 knockdown. Dexamethasone decreased $\beta$ arrestin 2 mRNA levels, but not $\beta$-arrestin1 levels. Finally, $\beta$-arrestin $1 \mathrm{mRNA}$ levels remained increased by $\beta$-arrestin 2 knockdown under dexamethasone treatments. Together, decreases in $\beta$-arrestin 2 expression directly may not contribute to increases in $\beta$-arrestin1 levels. Dexamethasone would not affect $\beta$-arrestin 1 gene levels.

Low $\beta$-arrestin expression and high SSTR2/ $\beta$-arrestin ratio reportedly correlate with responsiveness to longterm treatment with somatostatin analogues in patients with acromegaly [8]. In the present study, we found that SOM230 treatment increases $\beta$-arrestin1 level and does not alter $\beta$-arrestin 2 mRNA levels. SOM230 treatment could induce $\beta$-arrestin1 production. However, it would be unclear whether the SOM230-induced $\beta$-arrestin1 production inhibits SOM230-induced intracellular signals in the corticotroph tumor cells. On the other hand, $\beta$-arrestin2 knockdown increased basal Pomc mRNA level. These results suggest that $\beta$-arrestin2 mainly blocks ACTH synthesis, and may contribute to the inhibition of autonomous ACTH production in corticotroph tumor cells.

Cushing's disease is characterized by ACTH-dependent excessive circulating glucocorticoid concentrations. Glucocorticoids often affect receptor sensitivity or number. However, pretreatment with glucocorticoids fails to influence cell sensitivity to the inhibitory effect of SOM230 [3]. Given that SSTR5 is more resistant to glucocorticoids, the effects of SOM230 may last longer via the receptor. In the present study, treatment with dexamethasone, a kind of glucocorticoid, decreased $\beta$ arrestin2, but not $\beta$-arrestin1 mRNA levels. Therefore, in Cushing's disease, excessive cortisol production may inhibit $\beta$-arrestin2 synthesis. Under dexamethasone treatments, $\beta$-arrestin 2 knockdown-induced increases in Pomc mRNA levels were suppressed. Similarly, the $\beta$ arrestin2 knockdown-induced Sstr 5 mRNA levels were also inhibited under dexamethasone treatments. Excess glucocorticoids might be more potent factors to regulate

\section{A}

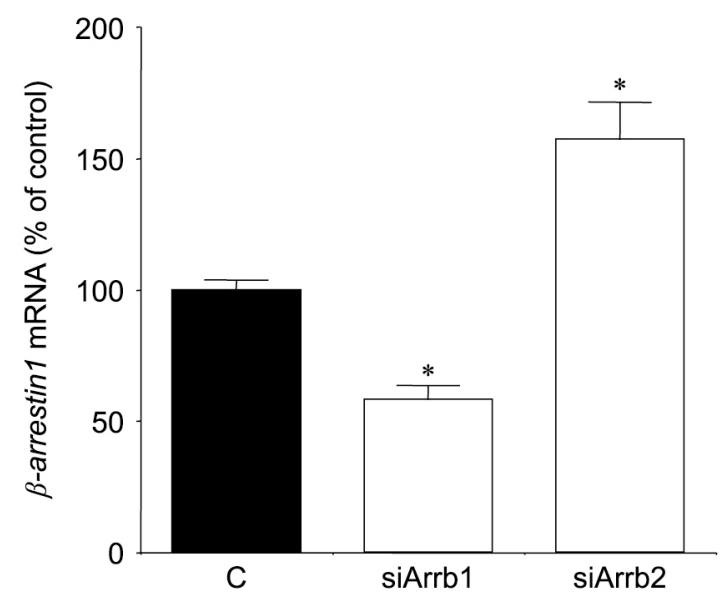

B

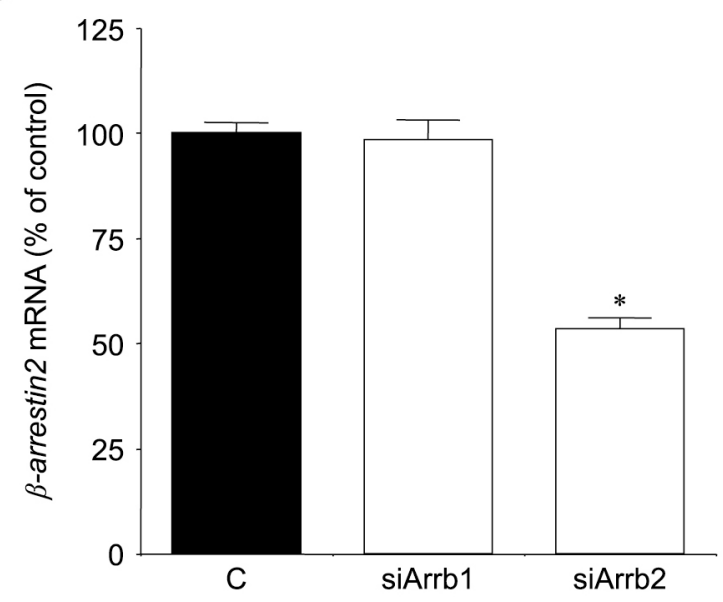

C

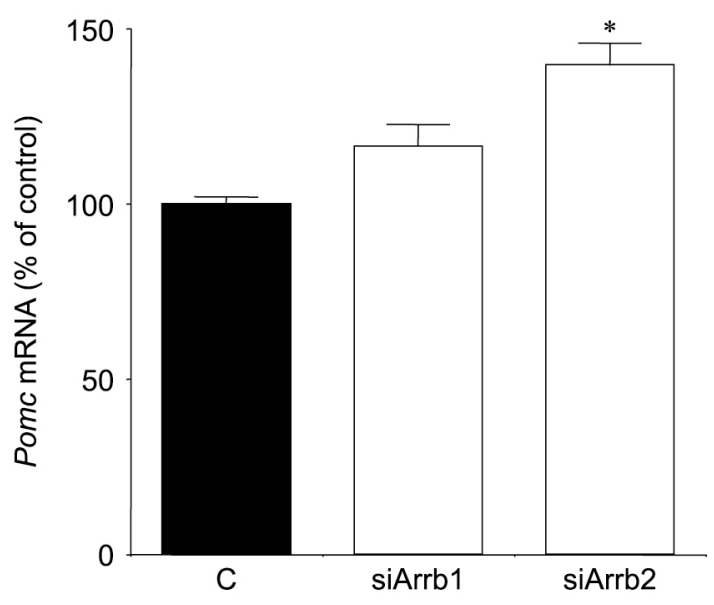

Fig. 3 Effects of $\beta$-arrestin $1 / 2$ on Pomc mRNA levels in murine corticotroph tumor cells. ${ }^{*} p<0.05$ [compared with control (C)]. (A) Effects of $\beta$-arrestin $1 / 2$ on $\beta$-arrestin 1 mRNA levels. (B) Effects of $\beta$-arrestin $1 / 2$ on $\beta$-arrestin 2 mRNA levels. (C) Effects of $\beta$-arrestin $1 / 2$ on Pomc mRNA levels. 

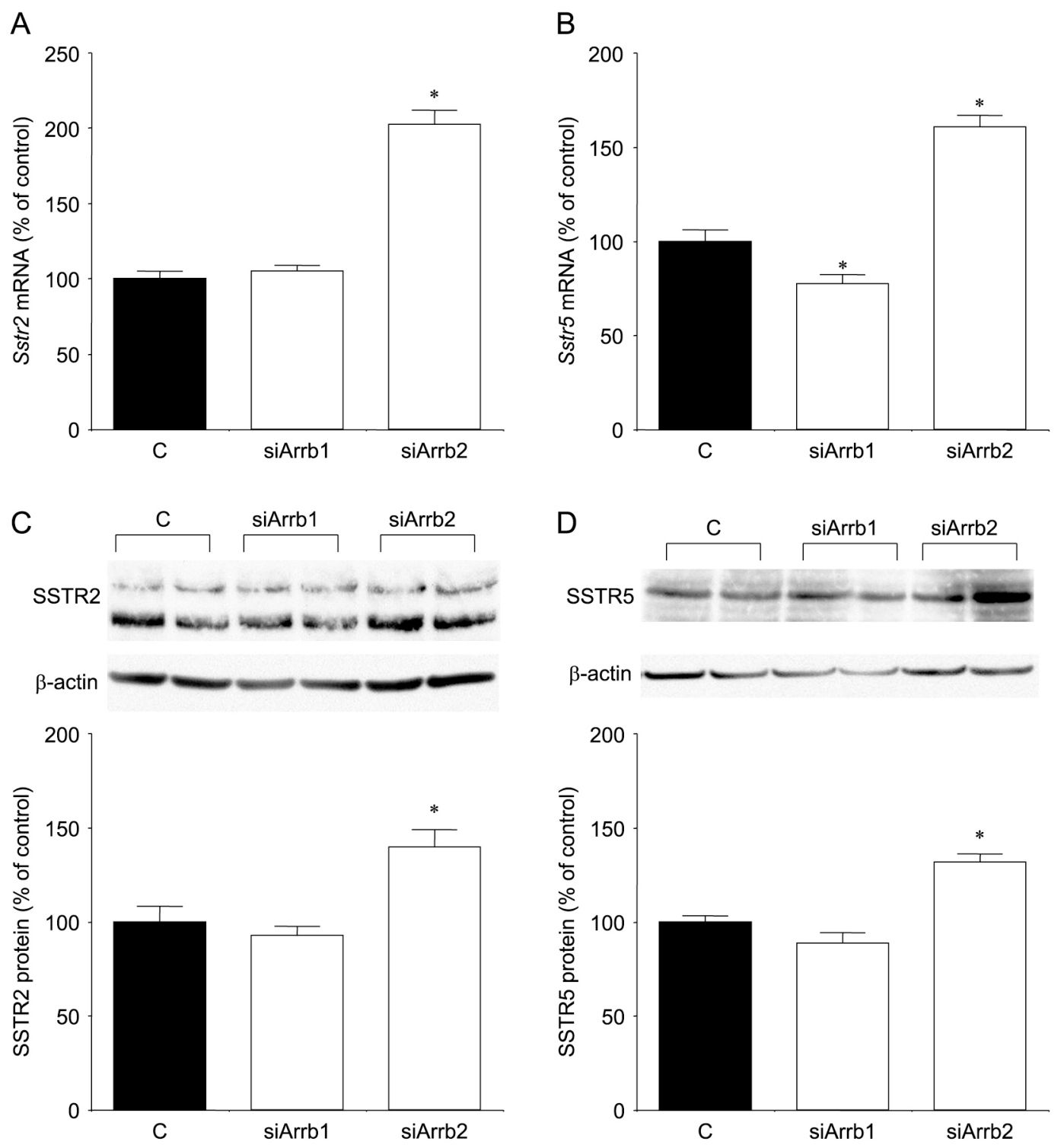

Fig. 4 Effects of $\beta$-arrestin1/2 on Sstr $2 / 5$ mRNA and their protein levels in murine corticotroph tumor cells. $*^{*}<0.05$ [compared with control (C)]. (A) Effects of $\beta$-arrestin1/2 on Sstr 2 mRNA levels. (B) Effects of $\beta$-arrestin1/2 on Sstr 5 mRNA levels. (C) Effects of $\beta$-arrestin1/2 on SSTR2 protein levels. A representative blot is shown. (D) Effects of $\beta$-arrestin1/2 on SSTR5 protein levels. A representative blot is shown.

expression of these genes in Cushing's disease.

Decrease in $\beta$-arrestin2 expression may diminish SSTR desensitization. $\beta$-arrestin 1 and $\beta$-arrestin2 are found to have distinct roles in GPCR signaling. For example, silencing of $\beta$-arrestin2 inhibits pituitary adenylate cyclase-activating polypeptide-induced extracellularsignal regulated kinase 1/2 activation, whereas, in contrast, silencing of $\beta$-arrestin1 increases its activation [14]. As in the pituitary adenylate cyclase-activating polypeptide signaling [14], $\beta$-arrestin 1 and $\beta$-arrestin 2 differentially regulate SSTR in corticotroph tumor cells. $\beta$ arrestin1 knockdown decreases Sstr 5 levels, whereas $\beta$ arrestin2 knockdown increases Sstr 2 and Sstr 5 and their protein levels. $\beta$-arrestin 2 knockdown might have a tendency to promote effects of SOM230 on Pomc mRNA levels, compared with the control or $\beta$-arrestin1 knockdown. Because decreased $\beta$-arrestin 2 expression mainly contributes to increases in SSTR2 and SSTR5 expression, it is possible to improve responses to somatostatin analogues in such conditions. However, the condition also may increase Pomc synthesis. In fact, our study shows that the $\beta$-arrestin knockdown-increased Pomc mRNA levels are canceled by SOM230 treatment. The effects of the $\beta$-arrestin knockdown should be examined in the future in vivo study.

Further study would shed light on this speculation. 


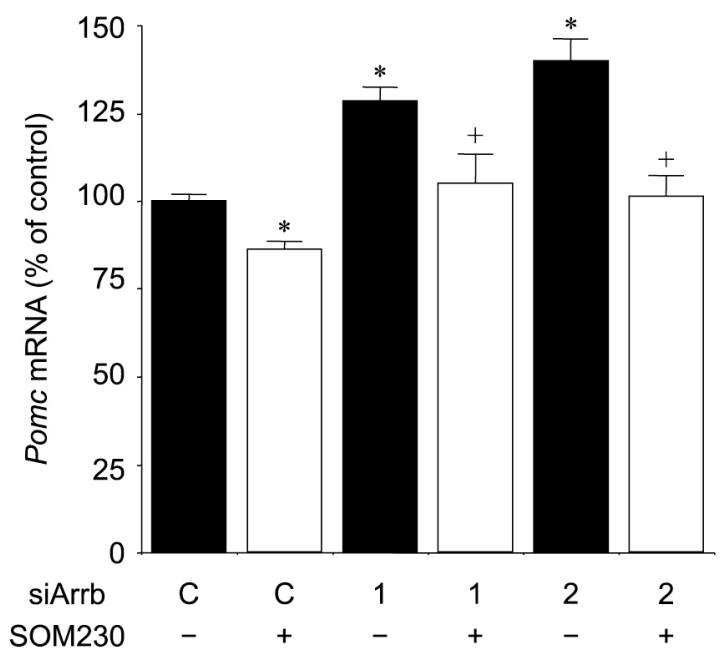

Fig. 5 Effects of $\beta$-arrestin $1 / 2$ and SOM 230 on Pomc mRNA levels in murine corticotroph tumor cells. ${ }^{*} p<0.05$ [compared with control siRNA (C) and SOM230 (-) group]. ${ }^{+} p<0.05$ [compared with each siRNA and SOM230 (-) group].

Furthermore, in this study, mouse corticotroph tumor cells were used. However, it is unclear whether these results are obtained in vivo or in human corticotroph tumor cells. For example, in the tumors of SOM230responded Cushing's disease, SSTR and $\beta$-arrestin2 expression levels might be increased and decreased, respectively. It should be explored to examine SSTR and $\beta$-arrestin expression levels in tumors of SOM230responded or SON230-resistance Cushing's disease. Studies to determine this are required in the future.

In conclusion, SOM230 treatment in corticotroph tumor cells promotes $\beta$-arrestin1 production. Decreased $\beta$-arrestin2 expression increases autonomous Pomc synthesis, and it also contributes to increases in both SSTR2 and SSTR5 expression levels. The $\beta$-arrestin2 knockdown-increased Pomc mRNA levels are canceled by SOM230 treatment.

\section{Funding}

This research was not funded through specific grants from any funding agencies in the public, commercial, or not-for-profit sectors.

\section{Disclosure of Potential Conflicts of Interest}

None of the authors have any potential conflicts of interest associated with this research.
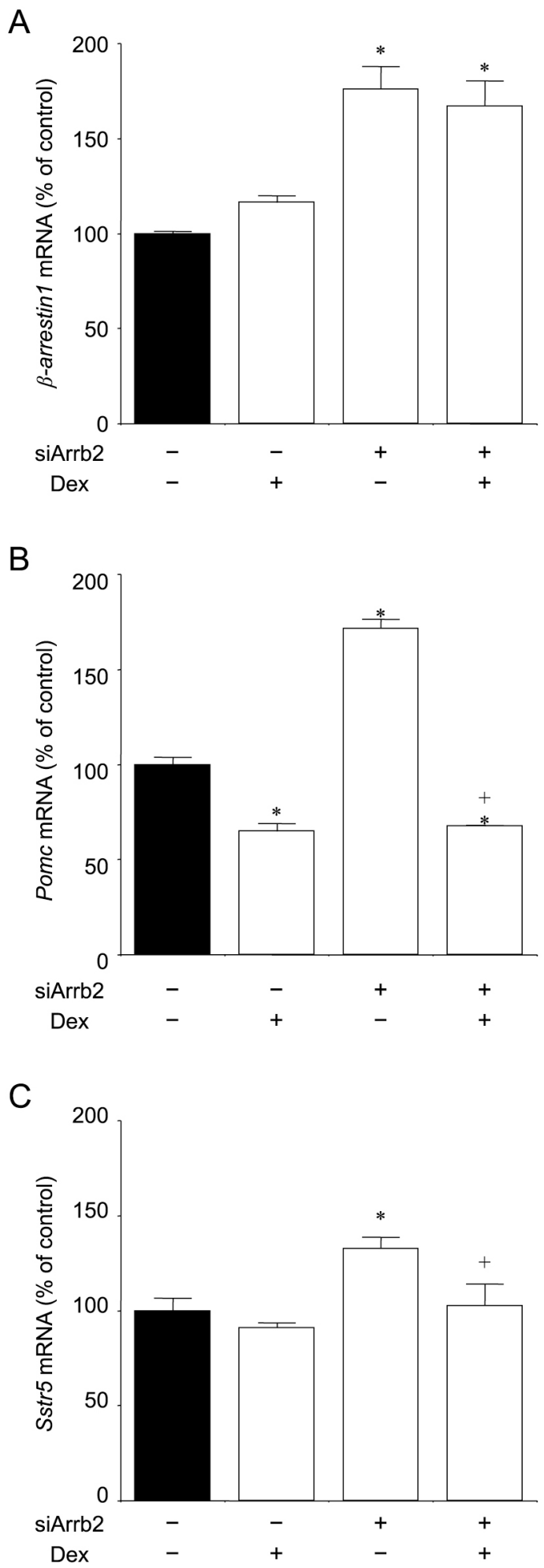

Fig. 6 Effects of $\beta$-arrestin2 and dexamethasone on $\beta$-arrestin1, Pomc, and Sstr 5 mRNA levels in murine corticotroph tumor cells. ${ }^{*} p<0.05$ [compared with siArrb2 $(-)$ and Dex (-) group]. ${ }^{+} p<0.05$ [compared with siArrb2 $(+)$ and Dex (-) group]. (A) Effects of $\beta$-arrestin2 and dexamethasone on $\beta$-arrestin1 mRNA levels. (B) Effects of $\beta$-arrestin2 and dexamethasone on Pomc mRNA levels. (C) Effects of $\beta$-arrestin2 and dexamethasone on Sstr 5 mRNA levels. 


\section{Compliance with Ethical Standards}

Human participants were not involved in this research.

\section{Authors' Contributions}

All authors participated in writing the manuscript and approved the final manuscript.

\section{References}

1. Gadelha MR, Wildemberg LE, Bronstein MD, Gatto F, Ferone D (2017) Somatostatin receptor ligands in the treatment of acromegaly. Pituitary 20: 100-108.

2. Bruns C, Lewis I, Briner U, Meno-Tetang G, Weckbecker G (2002) SOM230: a novel somatostatin peptidomimetic with broad somatotropin release inhibiting factor (SRIF) receptor binding and a unique antisecretory profile. Eur $J$ Endocrinol 146: 707-716.

3. Hofland LJ, van der Hoek J, Feelders R, van Aken MO, van Koetsveld PM, et al. (2005) The multi-ligand somatostatin analogue SOM230 inhibits ACTH secretion by cultured human corticotroph adenomas via somatostatin receptor type 5. Eur J Endocrinol 152: 645-654.

4. Hofland LJ (2008) Somatostatin and somatostatin receptors in Cushing's disease. Mol Cell Endocrinol 286: 199 205.

5. Feelders RA, de Bruin C, Pereira AM, Romijn JA, NeteaMaier RT, et al. (2010) Lamberts, Pasireotide alone or with cabergoline and ketoconazole in Cushing's disease. $N$ Engl J Med 362: 1846-1848.

6. Gatto F, Feelders R, van der Pas R, Kros JM, Dogan F, et al. (2013) $\beta$-Arrestin 1 and 2 and $G$ protein-coupled receptor kinase 2 expression in pituitary adenomas: role in the regulation of response to somatostatin analogue treatment in patients with acromegaly. Endocrinology 154: 47154725.

7. Cuevas-Ramos D, Fleseriu M (2014) Somatostatin receptor ligands and resistance to treatment in pituitary adenomas. J Mol Endocrinol v52: R223-R240.

8. Gatto F, Biermasz NR, Feelders RA, Kros JM, Dogan F, et al. (2016) Low beta-arrestin expression correlates with the responsiveness to long-term somatostatin analog treatment in acromegaly. Eur J Endocrinol 174: 651-662.

9. Ishigame N, Kageyama K, Takayasu S, Furumai K, Nakada Y, et al. (2016) Regulation of the expression of corticotropin-releasing factor gene by pyroglutamylated RFamide peptide in rat hypothalamic 4B cells. Endocr $J$ 63: 919-927.

10. Murasawa S, Kageyama K, Sugiyama A, Ishigame N, Niioka K, et al. (2014) Inhibitory effects of SOM230 on adrenocorticotropic hormone production and corticotroph tumor cell proliferation in vitro and in vivo. Mol Cell Endocrinol 394: 37-46.

11. Eigler T, Ben-Shlomo A (2014) Somatostatin system: molecular mechanisms regulating anterior pituitary hormones. J Mol Endocrinol 53: R1-R19.

12. Moriyama $\mathrm{T}$, Kageyama $\mathrm{K}$, Kasagi $\mathrm{Y}$, Iwasaki $\mathrm{Y}$, Nigawara $\mathrm{T}$, et al. (2005) Differential regulation of corticotropin-releasing factor receptor type 1 (CRF1 receptor) mRNA via protein kinase $\mathrm{A}$ and mitogenactivated protein kinase pathways in rat anterior pituitary cells. Mol Cell Endocrinol 243: 74-79.

13. Roosterman D, Brune NE, Kreuzer OJ, Feld M, Pauser S, et al. (2008) Intracellular degradation of somatostatin-14 following somatostatin-receptor3-mediated endocytosis in rat insulinoma cells. FEBS J 275: 4728-4739.

14. Shintani $Y$, Hayata-Takano A, Moriguchi K, Nakazawa T, Ago Y, et al. (2018) $\beta$-Arrestin1 and 2 differentially regulate PACAP-induced PAC1 receptor signaling and trafficking. PLoS One 13: e0196946. 